\title{
LAS EVALUACIONES EXTERNAS Y EL RENDIMIENTO ACADÉMICO DE LOS ALUMNOS COMO INSTRUMENTO DE MEJORA EDUCATIVA
}

\author{
Students" external assessments and academic performance as an \\ instrument for educational improvement
}

\author{
Eduardo López Bertomeo \\ e mail: edu_lopez_bertomeo@hotmail.com \\ Ángel Luis González Olivares \\ e-mail: aluisgonzalez@ucjc.edu \\ Universidad Camilo José Cela. España
}

\section{RESUMEN}

El presente artículo refleja la importancia que han adquirido las evaluaciones externas como instrumento de control, por parte de las Administraciones, del estado general de los sistemas educativos. Aunque es un tema de actualidad, la utilización de pruebas externas estandarizadas es tan antigua como los propios sistemas educativos, girando ya desde el inicio en torno al rendimiento académico de los alumnos. En la actualidad esas pruebas sirven de guía para la elaboración de leyes educativas que sin duda, están consiguiendo una auténtica renovación pedagógica en la que las instituciones educativas tratan de obtener los mejores resultados en el rendimiento de sus alumnos, suponiendo todo ello, la mejora general de los sistemas educativos.

PALABRAS CLAVE: política educacional; sistema educativo; evaluación de la educación; control de rendimiento escolar; calidad de la educación.

\section{ABSTRACT}

This article shows the importance of the external evaluation employed by the Administration as a tool to supervise the overall status of the educational system. Being a current issue, the use of a standardised external test is, however, as old as educational systems themselves which since their origin have always been related to students'academic performance. Currently, these tests serve as a guidance to elaborate educational laws that are undoubtedly acquiring an authentic pedagogical renewal through which educational institutions attempt to obtain the best results of their students' performance and, consequently, the general improvement of the educational system.

KEY WORDS: educational policy; educational systems; educational evaluation; achievement tests; educational quality.

Cómo referenciar este artículo / How to reference this article:

López Bertomeo, E. \& González Olivares, Á. L. (2018). Las evaluaciones externas y el rendimiento académico de los alumnos como instrumento de mejora educativa. Tendencias Pedagógicas, 31, 309-328. doi: http://dx.doi.org/10.15366/tp2018.31.017 


\section{CONSIDERACIONES PREVIAS}

España ha sido tradicionalmente un país con altos índices de fracaso escolar. Tanto es así que según los últimos datos del Instituto Nacional de Evaluación Educativa y Subdirección General de Estadística y Estudios del MECD (2016), la tasa de fracaso escolar y abandono temprano de la educación en el año 2015 se situaba en el $20 \%$, estando muy lejos del 11\% de la Unión Europea, siendo esto un claro indicador de que nuestro sistema educativo presenta evidentes posibilidades de mejora.

A partir de indicadores educativos como el citado, parece claro que podemos establecer una clara relación entre sociedad y sistema educativo, relación en la que encontramos como nexo de unión a las instituciones educativas, las cuales, en el cumplimiento del currículo oficial pretenden la formación integral de los alumnos, contribuyendo de esta forma al progreso de las sociedades en las que se contextualizan. En este sentido, parece lógico que esas sociedades reclamen cada vez mayor control e información sobre los contenidos que se transmiten y cómo se transmiten.

A este respecto, no ya solamente la Constitución Española reconoce que la educación es un derecho, sino que dentro de nuestra legislación educativa, la Ley Orgánica 8/1985, de 3 de julio, reguladora del derecho a la educación (LODE), recoge en su artículo 4.1, que padres o tutores, en relación a la educación de sus hijos o pupilos, tendrán entre otros, el derecho: "a) A que reciban una educación, con la máxima garantía de calidad, conforme con los fines establecidos en la Constitución, en el correspondiente Estatuto de Autonomía y en las leyes educativas"14.

Sobre esta base normativa, queda claro que las Administraciones educativas no solamente deben asegurar la educación de la sociedad, sino que también deben ofrecer un sistema educativo de calidad que dé respuesta a las exigencias de quienes representan, es decir, la sociedad en general y más concretamente los agentes educativos, en especial, alumnos y familias.

Así, considerando el carácter competitivo de la sociedad en la que nos encontramos, las demandas de familias y alumnos respecto a las instituciones educativas se concentran cada vez más en torno a un denominador común, la calidad educativa. De esta forma, son esas mismas familias y alumnos los que en la constante búsqueda de la excelencia educativa, reclaman con mayor énfasis cada vez, la consecución de mejores resultados académicos que les permita una mejor integración social y laboral, siendo las evaluaciones externas unos excelentes instrumentos de control y mejora del rendimiento académico de los alumnos, del funcionamiento y gestión de las instituciones educativas y en consecuencia, de la mejora constante de los sistemas educativos.

Así pues, desde una posición objetiva e independiente y lejos de controversias ideológicas, la aplicación de esas evaluaciones externas por parte de organismos de distinta naturaleza o de las Administraciones educativas, queda plenamente justificada siempre y cuando a través de ellas se pretenda rendir cuentas a la sociedad al tiempo que se genera debate social. Un debate que permita encontrar, tanto a los responsables de la política

${ }^{14}$ Texto consolidado a 30 de diciembre de 2013. 
educativa como a los principales agentes educativos, puntos de confluencia que favorezcan la consecución de un sistema educativo de calidad.

\section{SOCIEDAD Y EDUCACIÓN. POLÍTICA EDUCATIVA Y EVALUACIÓN.}

Las sociedades cambian y por ello, respecto a décadas pasadas, nos encontramos en una nueva dimensión política, económica, cultural, ética, moral, así como en un nuevo concepto de estilo de vida y relaciones interpersonales.

Ante tal cantidad de cambios sociales, destaca Tedesco (2009) que los desafíos que la educación debe afrontar son muy diferentes a los de épocas anteriores, entre otras razones porque en una sociedad de marcado carácter competitivo, el rol y el espacio que ocupa la educación, han cambiado. Consecuencia de ello, señala el propio autor, existe una permanente sensación de insatisfacción con lo que la educación puede ofrecer y por tanto, desde diversos grupos sociales se reclaman cambios más o menos profundos, ya sea en su estructura o en sus contenidos.

Ese grado generalizado de insatisfacción respecto a los sistemas educativos, nos ha dirigido a una situación en la que según autores como Longás et al. (2008, p.139), citando a Petrus (1998), existe en nuestra sociedad "una progresiva pérdida de confianza en la institución escolar, en parte porque la escuela no ha sido capaz de integrar los rápidos cambios producidos en la moderna sociedad, y también porque asistimos a un cambio de concepto de educación". Esto, continúan esos mismos autores, ha llevado a que en la actualidad las instituciones educativas sean víctimas de cierto rechazo e incomprensión hacia lo que en ellas acontece, provocando al mismo tiempo, que las propias escuelas muestren enormes reticencias a una efectiva relación con agentes educativos externos.

Obviamente nuestro país no es ajeno a esta situación descrita. Una situación que ha llevado a los responsables de nuestra política educativa a apostar por constantes cambios normativos, tratando con ello, de dar respuesta a una sociedad multicultural, intercultural, plenamente globalizada y por tanto, con fácil acceso a grandes cantidades de conocimientos. Así, como resultado del enorme y rápido desarrollo científico y tecnológico que estamos viviendo, las instituciones educativas, como bien señalan Longás et al. (2008, p. 139), han visto como a los ya tradicionales aprendizajes se han ido incorporando otros muchos, aspecto este que claramente "desborda las capacidades o los límites de intervención educativa de la escuela".

En este sentido, ya a finales del siglo pasado el expresidente de la Comisión Europea Jacques Delors (1996), señalaba que el siglo XXI ofrecería nuevas dimensiones de circulación y almacenamiento de informaciones y comunicaciones lo que conllevaría sin duda, en el ámbito educativo, la necesidad de alcanzar una nueva dimensión en la manera de transmitir una cantidad cada vez mayor de conocimientos que evolucionan rápidamente, por lo que como el propio Delors (1996) destacaba, la educación básica únicamente tendrá éxito si es capaz de establecer la estructura necesaria como para facilitar la adquisición de competencias en sus educandos. Competencias que les permita desarrollar la autonomía suficiente como para hacer efectivo el aprendizaje a lo largo de la vida. 
En cualquier caso, en la escuela actual, alcanzar esa situación descrita no es tarea sencilla, ya que si bien es cierto que los sistemas educativos de los países desarrollados han evolucionado enormemente en las últimas décadas, también es verdad que el carácter innovador y globalizado de nuestra sociedad hace que sea "menor la capacidad de cambio del sistema educativo respecto al sistema social” (Esteve, 2009, p. 2).

Dentro de ese proceso de adaptación de los sistemas educativos, los grandes organismos internacionales y supranacionales que surgieron, la mayoría de ellos, a partir de la finalización de la Segunda Guerra Mundial, tienen un protagonismo cada vez mayor en el desarrollo de acciones y propuestas de carácter educativo, provocando "una sutil pero perceptible armonización en las formas de entender las grandes cuestiones que afectan a la política educativa" (Valle, 2012, p.112). De hecho, en el caso de nuestro país, estamos de acuerdo en afirmar que "hoy se puede decir que más de la mitad de las políticas educativas que tradicionalmente han tenido un carácter doméstico o nacional se deciden en Bruselas" (De Puelles, 2010, p. 56)

Así, aunque resulta evidente que esos organismos no tienen competencias en materia de ordenación u organización de los sistemas educativos nacionales, sí que influyen, tal y como afirma Valle (2012), sobre las políticas educativas nacionales en cuanto a propuestas, recomendaciones, planes de acción, trabajo o cooperación, siendo aquí, en este contexto de influencia transnacional donde, desde nuestro punto de vista, la dimensión política de la evaluación cobra especial relevancia a través de las evaluaciones externas. ${ }^{15}$ Unas evaluaciones que se orientan principalmente al análisis del rendimiento académico de los alumnos como una de las dimensiones que mejor definen el estado o funcionamiento de los sistemas educativos. Tal es así que, como bien señalan Calero y Choi (2012), en las últimas décadas el interés por analizar el grado de adquisición de conocimientos y destrezas por parte del alumnado a través de evaluaciones externas, ha pasado a desempeñar un papel protagonista entre los instrumentos utilizados en política educativa.

\section{DIMENSIÓN POLÍTICA DE LAS EVALUACIONES EXTERNAS.}

\subsection{Las evaluaciones externas en el siglo XIX.}

En términos generales podríamos decir que, previo a la configuración de los primeros sistemas educativos, históricamente no existió nunca demasiado interés por parte de los Estados en la formación básica de las clases más desfavorecidas, dejándose esa labor en manos de la Iglesia o de instituciones locales. Esta situación era totalmente contraria en el contexto de las enseñanzas universitarias, ya que como indica De Puelles (2010, p. 212),

\footnotetext{
${ }^{15}$ Gran parte de las propuestas y recomendaciones de los grandes organismos internacionales y supranacionales, en materia educativa, se estructuran a partir de pruebas de evaluación externa. Ejemplo de ello son PISA, PIAAC o TALIS de la OCDE; las pruebas EECL de la Unión Europea o las pruebas PIRLS y TIMMS de la IEA (Asociación Internacional para la Evaluación del Aprovechamiento Escolar). Más información sobre pruebas externas de carácter internacional en http://www.mecd.gob.es/inee/publicaciones/estudios-internacionales.html
} 
esas enseñanzas tenían gran "incidencia en la formación de los cuadros dirigentes, y por tanto, en el reclutamiento de la burocracia estatal".

Sin embargo, el punto de inflexión a partir del cual los diferentes Gobiernos van a empezar a preocuparse por la formación básica o elemental de los ciudadanos, señala el mismo De Puelles (2010, p. 232) estuvo en el firme interés por parte de los Estados en desempeñar un papel protagonista en el nacimiento de los primeros sistemas educativos del siglo XIX, sistemas que "se convirtieron de este modo en instituciones que formaron parte del aparato estatal, cumpliendo funciones diversas de acuerdo con las variables necesidades nacionales". ${ }^{16}$

Sin ser nuestro país una excepción, en ese contexto de implicación de los Gobiernos en la formación y desarrollo de los sistemas educativos, parece lógico pensar que ya desde el inicio, las Administraciones educativas requiriesen de instrumentos o mecanismos de evaluación que las permitiera adaptar o mejorar esos sistemas a través del seguimiento y control de los principales elementos que lo componen: currículos, rendimiento académico del alumnado, eficacia de los centros educativos o la propia actividad docente.

En este sentido, estamos de acuerdo con De la Orden (2009) en señalar que la evaluación, realizada con rigor sistemático, ofrece como principal objetivo dar un determinado valor a todos los campos sociales que se consideran importantes, siendo sin duda uno de ellos, la educación. Así pues, desde la perspectiva educativa, el propio De la Orden (1997b), define la evaluación como:

El proceso sistemático de recogida, análisis e interpretación de información relevante y fiable para describir cualquier faceta de la educación y formular un juicio de valor sobre su adecuación a un criterio, que represente un valor aceptado, como base para la toma de las oportunas decisiones optimizantes de dicha faceta.

(De la Orden, 2009, p. 23).

De esta forma, considerando que en el ámbito educativo cualquier variable puede ser objeto de evaluación, ya desde el mismo momento en el que los primeros Estados modernos comenzaron a canalizar sus políticas educativas a través de una compleja base legislativa, la evaluación del rendimiento académico de los alumnos mediante pruebas externas como instrumento para la mejora de los sistemas educativos, fue un tema incipiente que poco a poco ha permitido, como bien indica Rodríguez (2010), la consolidación de profundas raíces políticas.

Así, si tenemos en cuenta los principales textos educativos de carácter legislativo, en el caso de nuestro país, hubo que esperar hasta 1821 para que se aprobase la primera ley general de educación. Fue el Reglamento General de Instrucción Pública, a través del cual, se reguló "la enseñanza estatal, pública, uniforme y gratuita, sentando las bases de los sucesivos sistemas educativos" (Santamaría, 2007, p. 167).

\footnotetext{
${ }^{16}$ Entre ellos y principalmente, la idea de nación.
} 
En el citado Reglamento, que por las convulsas circunstancias políticas no llegó a aplicarse, ${ }^{17}$ aparecen ya los primeros indicios de una evaluación externa entendida como instrumento de mejora de algunos de los principales componentes del sistema educativo de la época, en este caso, la labor de maestros y el aprendizaje de los alumnos. Así, en el artículo 31 del citado Reglamento se recoge que: "Además de los exámenes particulares que sufran los discípulos en su respectiva clase, se celebrarán todos los años exámenes públicos, con asistencia de las Autoridades provinciales, para promover por este medio la aplicación de los maestros y discípulos". Asimismo, como aparece en el artículo 101 y entre otras cosas, para velar por la efectiva mejora de los métodos de enseñanza, se crea lo que será el germen de los actuales servicios de Inspección educativa, la Dirección General de Estudios.

Posteriormente, ya en la Década Absolutista (1823-1833), se aprueba en 1825 el Plan y Reglamento de Escuelas de Primeras Letras. En este Plan se establecía que los alumnos fueran evaluados en exámenes públicos "según sus clases en todos los ramos que comprende la primera educación, haciéndoles las preguntas con claridad y sencillez" (art. 76), otorgándose premios a los más avanzados (art. 84). Asimismo, la antigua Dirección General de Estudios se transformó, según nos explica Real (2012), en la Junta Superior de Inspección, la cual, se articulaba en las Juntas de Capital y Juntas de Pueblo, contando entre sus funciones, no solamente la de asistir a esos exámenes públicos (art. 75), sino también proponer los medios necesarios para la mejora de los procesos de enseñanza (art. 133 y 145).

Dentro del mismo orden político y orientado más a lo que podría ser la enseñanza secundaria, en 1826 se aprueba el Reglamento General de las Escuelas de Latinidad y Colegios de Humanidades, en la que además de incidir en la cuestión de las evaluaciones generales y públicas a los alumnos (art. 34), la Junta Superior de Inspección es sustituida por la Inspección General de Instrucción Pública (art.104).

Este nuevo órgano de control educativo, iba a tener una gran importancia en el inicio y consolidación del uso estadístico de datos e informes de evaluación como instrumento de mejora del sistema educativo, ya que, como destaca López del Castillo (2013), siguiendo el precedente de la Junta Superior de Inspección, la nueva Inspección General se ocupó de la recogida de información estadística, publicándose en un primer momento la relativa a la enseñanza secundaria y superior y no siendo hasta 1830, cuando se valoró la necesidad de organizar de manera sistematizada la recogida de datos en todas las etapas educativas, añadiéndose en algunos casos, notas de los alumnos relativas a su capacidad, esfuerzo y comportamiento. ${ }^{18}$ Este hecho facilitó el análisis y mejora de nuestro sistema educativo, ya que permitió mayor fiabilidad a la Inspección General en el cumplimiento de algunos de sus cometidos, entre

\footnotetext{
${ }^{17}$ El Reglamento General de Instrucción Pública se elabora en el llamado Trienio Liberal (18201823) e inmediatamente después, sin tiempo para su aplicación, entramos en la Década Absolutista (1823-1833).

${ }^{18}$ Según López del Castillo (2013, p. 86), todos esos datos estadísticos fueron publicados en la Gaceta de 23 de febrero de 1833, lo que supuso en nuestro país, al contener datos relativos a los diversos niveles de enseñanza, "la primera estadística general relativa a la Instrucción pública".
} 
ellos, según la propia López del Castillo (2013), informar del estado de las escuelas y de sus progresos en los procesos de enseñanza.

Como puede observarse, en esa relación que se establece ya desde el principio entre evaluaciones externas y mejora del sistema educativo en sus diferentes vertientes, ${ }^{19}$ la recogida de datos estadísticos empieza a cobrar especial relevancia. Esta dinámica tuvo su continuidad finalizado el periodo absolutista antes reseñado. España entraba en una etapa política de marcado carácter liberal, no exenta de tensiones políticas, con altas tasas de analfabetismo entre su población. Por ello, como nos viene a señalar Santamaría (2007), gran parte de los esfuerzos realizados en la política educativa de la época, partieron de la consideración de la educación como instrumento ideal para la alfabetización de la ciudadanía y por tanto, como un eficaz mecanismo para el progreso económico y social de nuestro país. Este hecho, conllevó la aprobación de diversos textos educativos de carácter legislativo, en cuanto a Leyes y Reglamentos, en los que, además de regular el proceso de instrucción y evaluación del alumnado y considerar también la adecuada preparación de los maestros, se incidía en la recogida de datos como instrumento de mejora educativa en los diversos niveles de enseñanza.

Tal es el caso, en 1836, del Plan General de Instrucción Pública o Plan Duque de Rivas, que con poco más de una semana de vigencia, estableció en su articulado una Comisión de Instrucción Pública encargada entre otras de "proporcionar al Gobierno todos los datos que le pida sobre la enseñanza, y formar la estadística anual, así del número de alumnos que asistan a las escuelas primarias, Institutos o Universidades, como de los fondos de estos establecimientos" (art.116, apdo.10).

Posteriormente, en el contexto de la nueva Constitución de 1837, el 26 de noviembre de 1838 es aprobado el Reglamento Provisional de las Escuelas Públicas de Instrucción Primaria Elemental, más conocido como Reglamento Montesino. En el contexto de las evaluaciones, además de los exámenes habituales en las aulas, se establecían evaluaciones generales en los que los alumnos eran "examinados por secciones en las diferentes clases o ramos de enseñanza, haciéndoles preguntas claras, pero no determinadas o estudiadas precisamente para el acto" (art. 87). Especialmente interesante resulta el artículo 88 del Reglamento, por ser la primera gran referencia a la evaluación de los alumnos como factor para determinar el nivel educativo de las escuelas: "La Comisión local comunicará á la provincial el juicio que hubiere formado, á consecuencia del examen, de los progresos de la Escuela".

Por otro lado, después de un intenso periodo de constantes cambios legislativos en materia educativa llegamos a 1857, año de aprobación de la Ley de Instrucción Pública de 9 septiembre de 1857, conocida como Ley Moyano. Dentro de un contexto político liberal, esta Ley, consagraba una tipología de evaluación general y pública ya presente en Leyes precedentes a lo que se añadió, en materia de control y mejora educativa, la creación del Real Consejo de Instrucción Pública, el cual tenía competencias "en la revisión de los programas de enseñanza, y en las modificaciones que en ellos se hicieren" (art.

\footnotetext{
${ }^{19}$ En nuestra opinión, además del rendimiento escolar de los alumnos, otra de esas vertientes se refiere al nivel de las instituciones escolares y profesores, la cual está directamente relacionada con la primera.
} 
256, apartado quinto). Asimismo, a nivel local, la Ley Moyano establece las Juntas de Instrucción Pública, entre cuyas competencias encontramos la de "promover las mejoras y adelantos de los Establecimientos de primera y segunda enseñanza" (art. 286, apartado segundo), así como elaborar un registro de maestros en los que se incluyan, entre otros datos, los resultados obtenidos en el proceso de enseñanza (disposición provisional 9âa).

Por último, no sin la aparición de movimientos que apostaban por corrientes pedagógicas más innovadoras que las propuestas por la Ley Moyano, la Institución Libre de Enseñanza entre ellas (Rodríguez, 2010), destaca Real (2012) que será esta Ley la que proporcione estabilidad y continuidad a nuestro sistema educativo de forma duradera, siendo la principal razón el hecho de encajar "con las características económicas, sociales y políticas de la sociedad española" (De Puelles, 2008, p. 10). De esta forma, no será hasta la Ley General de Educación de 1970 cuando se pueda hablar de otra de las grandes Reformas del sistema educativo en nuestro país. Reforma que supondría un punto de inflexión en nuestro sistema educativo, introduciendo, entre otros aspectos, una nueva terminología en materia de evaluación.

\subsection{Las evaluaciones externas en el siglo $\mathrm{XX}$}

\subsubsection{Transición educativa}

La Ley Moyano gobernó el contexto educativo de nuestro país durante más de un siglo. A lo largo de esos años se van a producir múltiples reformas pero, como indica De Puelles (2008), la estructura del sistema educativo español permanecería inalterada hasta 1970, año de aprobación de la Ley 14/1970, de 4 de agosto, General de Educación y Financiamiento de la Reforma Educativa (LGE).

En 1970 nuestro país mostraba altos índices de desarrollo social, índices que daban muestra del paso de una sociedad rural a una sociedad industrial agrupada en grandes núcleos urbanos, índices, en definitiva, que permitieron a nivel internacional poder hablar del milagro económico español. Esta situación favoreció que dentro del Régimen dictatorial que vivía nuestro país, al menos a nivel educativo, se impusieran, según nos explica Cabrera (2007), los sectores gubernamentales menos reaccionarios, logrando por tanto mayor aceptación por parte de la comunidad internacional. En este contexto social y económico, la LGE o Ley Villar Palasí indica el propio Cabrera (2007), fue en cierto modo contradictoria, ya que fue una Ley cuya elaboración y en parte aplicación se situaron en un periodo dictatorial, confluyendo en ella al mismo tiempo, la mayoría de las tendencias pedagógicas más innovadoras de la época. Esto supuso que con el devenir educativo de nuestro país, la LGE se constituyera en la base a partir de la cual se sustentaron todas las novedades legislativas posteriores.

En cualquier caso, en el ámbito de evaluación, la nueva LGE introduce por primera vez en una gran Ley que trata de estructurar y regular todo el sistema educativo de nuestro país, nueva terminología. Terminología que hacía mención explícita al rendimiento escolar o educativo y que supuso los primeros pasos para que en materia de evaluación se fueran imponiendo las políticas 
educativas de los países de nuestro entorno. De hecho, la LGE de 1970 es "acreditada por expertos internacionales, pertenecientes a organismos que tienen como principal función orientar políticas internacionales a favor de la normalización económica, social y política del capitalismo (OCDE, UNESCO)" (Cabrera, 2007, p. 162).

De esta forma, en sintonía con esas tendencias internacionales, en la LGE se establecía una relación directa entre el rendimiento académico de los alumnos y la eficacia de los centros a través de la acción docente. En este sentido, es el artículo 11.1 el que establece que: "La valoración del rendimiento educativo se referirá tanto al aprovechamiento del alumno como a la acción de los Centros" (p. 12528). Profundizando en este aspecto, en el punto 5 del citado artículo 11 se hace referencia a que el rendimiento de los Centros educativos se valorará atendiendo, entre otras cosas, al rendimiento de los alumnos tanto en su vida académica como profesional o a la aplicación de métodos innovadores de enseñanza. En esta línea de actuación, para asegurar el cumplimiento legislativo y en sintonía con otros textos legislativos anteriores, en la LGE de 1970 se crea un Servicio de Inspección Técnica de Educación que se ocupará, según lo establecido en el artículo 142.1.d de "evaluar el rendimiento educativo de los Centros docentes y Profesores" (p. 12542).

Posteriormente, pasados exactamente veinte años de aquella LGE y ya con cierta madurez democrática, en nuestro país se hacía necesaria una nueva Ley educativa que rompiese definitivamente con el periodo previo a la Carta Magna de 1978. En ese contexto nace en 1990 una Ley que fue más allá de lo educativo y que tuvo por tanto, auténtico calado social. Nos referimos a la Ley Orgánica 1/1990, de 3 de octubre de 1990, de Ordenación General del Sistema Educativo, la LOGSE. ${ }^{20}$

Es cierto que hasta la aprobación de esta Ley se formularon y aprobaron muchas otras leyes que trataban de ajustar el sistema educativo heredado de la LGE de 1970 al nuevo orden constitucional surgido en 1978. Sin embargo, en nuestra opinión, fue la LOGSE la que puso fin a un periodo de Transición educativa, ya que como destaca Cabrera (2007, p.166), fue "percibida como el punto de llegada de un largo proceso hacia la renovación de la educación en España". Además, la LOGSE supuso la necesidad de ajustarse a los grandes cambios culturales, políticos, económicos y sociales que se estaban produciendo en nuestro país, de hecho, como bien señala De Puelles (2008, p. 12) en los albores de los años 90 nos encontrábamos con una sociedad que "se encaminaba ya por la vía de la información y el conocimiento".

En el ámbito de la evaluación, la LOGSE "diluye la importancia del rendimiento académico frente a la relevancia creciente de las habilidades, las actitudes, el progreso del alumno, etc." (Cabrera, 2007, p. 180). No obstante, desde la perspectiva de la evaluación como instrumento de mejora educativa, uno de los ejes más renovadores de la LOGSE fue el de la calidad de la enseñanza, la cual, como indica De Puelles (2012), sería incluida por primera vez en la historia educativa de nuestro país como un título específico. Así, en el

\footnotetext{
${ }^{20}$ La LOGSE fue elaborada y aprobada bajo la amplia mayoría parlamentaria del PSOE y entendiéndose, según Cabrera (2007) por una buena parte de la izquierda ideológica como una ruptura respecto al pasado educativo.
} 
artículo 62.1 de la propia Ley, se establece que la evaluación del sistema educativo "se aplicará sobre los alumnos, el profesorado, los centros, los procesos educativos y sobre la propia Administración" (p. 28936). Además, tal y como recuerda Cabrera (2007), la LOGSE introdujo una importante novedad en cuanto a institucionalizar la evaluación a través de la creación del Instituto Nacional de Calidad y Evaluación, cuyas funciones quedaron recogidas en el artículo 62.4, siendo:

a) Elaborar sistemas de evaluación para las diferentes enseñanzas reguladas en la presente ley y sus correspondientes centros.

b) Realizar investigaciones, estudios y evaluaciones del sistema educativo y, en general, proponer a las Administraciones educativas cuantas iniciativas y sugerencias puedan contribuir a favorecer la calidad y mejora de la enseñanza (p. 28937).

Por otro lado, si bien la LOGSE no prestó demasiada atención al ámbito de las evaluaciones externas como instrumento de medida del rendimiento académico de los alumnos, es destacable que tratando de corregir algunas de sus deficiencias se aprobó, con la mayoritaria oposición del profesorado, la Ley Orgánica 9/1995, de 20 de noviembre, de la participación, la evaluación y el gobierno de los centros docentes (LOPEG).

La LOPEG estuvo vigente hasta el año 2006 y como novedad respecto a la LOGSE, encontramos ya, en su artículo 29.2, el término evaluación externa. Estas evaluaciones se referían a los centros docentes y en virtud del artículo 29.4, no solamente a cuestiones organizativas o de funcionamiento y gestión, sino también a la totalidad de las actividades que conforman los procesos de enseñanza y aprendizaje que en ellos se desempeñan. Además, en ese mismo artículo se recogía que la Administración informaría a la comunidad educativa de los resultados obtenidos, instando a los propios centros a resolver las carencias detectadas.

\subsubsection{Una nueva etapa en las evaluaciones externas.}

En el año 2002 y no sin dificultades, el partido en el Gobierno saca adelante la Ley Orgánica 10/2002, de 23 de diciembre, de Calidad de la Educación (LOCE).$^{21}$ Como dijimos con anterioridad, la LOGSE presentaba algunos errores, principalmente en lo relacionado con la promoción de curso, siendo ahí donde la LOCE trató de poner mayor énfasis. De esta forma, bajo la justificación de altas tasas de abandono escolar y bajos niveles en el rendimiento académico de los alumnos (especialmente en Secundaria) detectados por evaluaciones nacionales e internacionales, así como a las necesidades derivadas de la plena integración de España en el entorno europeo, la Ley se configuró, tal y como se recoge en su Exposición de Motivos, en torno a varios ejes, entre ellos, la orientación del sistema educativo hacia la obtención de resultados mediante evaluaciones a alumnos, profesores y centros educativos, otorgando a estos últimos, altos niveles de responsabilidad en la consecución de adecuados niveles de rendimiento entre el alumnado.

\footnotetext{
${ }^{21}$ La LOCE se elabora y aprueba en la segunda legislatura del Gobierno liderado por el Partido Popular (2000-2004).
} 
El poco consenso social y profesional con el que contó esta ley hizo que con la llegada de un nuevo Gobierno de diferente ideología, en el año 2004 se paralizase el calendario de aplicación de la LOCE y se aprobase en el año 2006 la Ley Orgánica 2/2006, de 3 de mayo, de Educación (LOE), la cual, según se recoge ya en su Preámbulo es presidida por tres principios fundamentales: proporcionar una educación de calidad, la implicación de todos los componentes de la comunidad educativa para alcanzar ese objetivo de calidad y la convergencia con los objetivos propuestos por la Unión Europea.

De esta forma, destaca Fernández (2008) que con la elaboración y aprobación de la LOE se trató de no radicalizar ninguna de la dualidad educativa que había caracterizado históricamente a nuestro país, aspecto que también se refleja en lo que concierne al análisis del rendimiento académico de los alumnos como instrumento de mejora del sistema educativo. De hecho, siendo la LOE una Ley de corte progresista recoge aspectos de la derogada LOCE, entre ellos, las evaluaciones de diagnóstico. Este hecho, favoreció que nuestro sistema educativo entrase en una nueva dimensión respecto al análisis del rendimiento académico de los alumnos mediante evaluaciones externas. Para ello, haciéndose nuevamente eco de la LOCE, la nueva Ley introdujo un nuevo concepto curricular, las competencias básicas, ${ }^{22}$ las cuales se constituirían en el eje vertebrador de las ya citadas evaluaciones de diagnóstico. Estas evaluaciones, según se recogía en el Preámbulo de la propia Ley, tendrían un valor formativo y orientador para los centros docentes e informativo para la comunidad educativa sobre la situación de alumnos y centros, así como del funcionamiento del propio sistema educativo. Además, esas evaluaciones externas permitirían analizar el nivel de adquisición de las competencias básicas de los alumnos y se realizarían por todos los centros a los alumnos de $4^{\circ}$ de Primaria (art. 21) y 2o de Educación Secundaria Obligatoria (art. 29).

En cualquier caso, con el cambio de Gobierno en el año 2011 y nuevamente bajo el paraguas de los profundos cambios sociales a los que nuestra sociedad debe enfrentarse, en el año 2013 de aprueba la Ley Orgánica 8/2013, de 9 de diciembre, para la mejora de la calidad educativa (LOMCE), una Ley que no deroga la anterior, sino que la modifica. Además, tal y como aparece en su Preámbulo, en las razones que justifican la elaboración de la LOMCE cobran gran importancia, como ya sucedía en la LOCE, las evaluaciones externas de carácter internacional, principalmente el Informe PISA del año 2009 y los datos de EUROSTAT, los cuales presentaban datos educativos que mostraban como nuestro país se encontraba por debajo de las medias internacionales, lo cual, dificultaba la convergencia con los grandes objetivos sobre calidad educativa marcados por la Unión Europea. Todo esto llevó a la Administración a determinar que nuestro sistema educativo tenía un gran margen de mejora y que para ello, debía apostar decididamente por unas evaluaciones externas que, con efecto formativo, diagnóstico y académico en algunos casos, valorasen el rendimiento académico de los alumnos al final de

\footnotetext{
${ }^{22}$ En la normativa que desarrollaba la LOE, como por ejemplo el Real Decreto 1513/2006, de 7 de diciembre, por el que se establecían las enseñanzas mínimas de la Educación Primaria, en su Preámbulo, las competencias básicas eran definidas como aprendizajes imprescindibles para la posterior aplicación de saberes adquiridos.
} 
cada etapa educativa ${ }^{23}$ permitiendo, según se recoge en la propia Ley y en función de los resultados obtenidos, establecer las medidas necesarias como para mejorar el nivel de los aprendizajes de los alumnos, las medidas de gestión de los centros o las políticas educativas de las Administraciones y en definitiva, la calidad del sistema educativo.

\subsubsection{Dimensión autonómica de las evaluaciones externas.}

Como hemos visto, en las políticas educativas imperantes a nivel internacional, orientadas en gran medida a la mejora de la calidad educativa por medio de evaluaciones externas individualizadas del rendimiento académico de los alumnos, nuestro país no es en absoluto ajeno. No es ajeno ni desde el punto de vista de la Administración central ni desde el punto de vista autonómico.

De esta forma, en el contexto de un país descentralizado a nivel educativo como el nuestro, las Comunidades Autónomas presentan un nivel competencial suficiente como para establecer pruebas de evaluación externa entre sus alumnos. En este sentido, es destacable por innovador el caso de la Comunidad de Madrid, la cual a partir de diversos informes internos que mostraban las carencias de los alumnos en las áreas curriculares de Lengua y Matemáticas en su paso de Primaria a Secundaria, decidió ya en el año 2005 la realización de las denominadas pruebas CDI (Conocimientos y Destrezas Indispensables) entre los alumnos de $6^{\circ}$ de Primaria. Los resultados nada positivos de estas pruebas llevaron a la Administración autonómica a la tramitación de un Plan General de Mejora de las Destrezas Indispensables. ${ }^{24}$ Un Plan, que en función de la normativa que lo regulaba, orientaba la política educativa de la región hacia la mejora de los resultados académicos de sus alumnos mediante medidas tales como la realización anual de las pruebas externas ${ }^{25}$ o la publicación de estándares de aprendizaje en Lengua y Matemáticas que se debían alcanzar en los diferentes ciclos en los que se estructuraba la etapa de Primaria. Además, según la normativa que regulaba las pruebas CDI tanto en Primaria como en Secundaria, se hacía alusión a que una vez analizados los resultados obtenidos por los centros, se debía elaborar un Plan de Mejora a través del cual se reforzarían o corregirían diversos aspectos de carácter organizativo o didáctico para la mejora de los resultados en años sucesivos. De hecho, para que ese plan tuviese efecto anual, se debía incluir en la Programación General Anual.

\section{EL VALOR ACTUAL DE LAS EVALUACIONES EXTERNAS}

\footnotetext{
${ }^{23}$ El artículo 20.3 de la LOE, a partir de la reforma operada por la LOMCE, establece también una evaluación individualizada y externa a los alumnos de 3 de Primaria.

${ }^{24}$ Regulado en la Orden 5420-01/2005, de 18 de octubre, del Consejero de Educación, por el que se aprueba el Plan General de Mejora de las Destrezas Indispensables. Allí, también se daba cuenta de que aproximadamente el $30 \%$ de los alumnos de Primaria no tenían los conocimientos mínimos en Lengua y Matemáticas como para cursar exitosamente la etapa de Secundaria.

${ }^{25}$ En el año 2008 las pruebas de evaluación externa CDI se ampliaron a 3o de ESO y 1ำ de Diversificación Curricular.
} 
En la actualidad nos encontramos con una sociedad que cambia, que evoluciona a velocidad vertiginosa y en consecuencia, en las últimas décadas, en el ámbito de la educación como parte integrante de esa sociedad, parece que nos hemos situado en una auténtica revolución educativa. Una revolución en la que la evaluación y más concretamente una de sus dimensiones, las evaluaciones externas, desempeñan un papel protagonista como motor de cambio y progreso.

La presencia de evaluaciones externas como factor de mejora educativa, han sido prácticamente una constante en los diversos textos legislativos que se han ido sucediendo a lo largo del tiempo. No obstante, hoy día las evaluaciones externas están plenamente de actualidad y parte de su éxito, señalan Calero y Choi (2012, p. 30), "ha estado basado en el impacto mediático de los programas internacionales de evaluación de competencias y, más en concreto, del programa PISA de la OCDE”. Además, en gran medida, esa relevancia se debe también a un cambio de mentalidad en la comunidad educativa, especialmente en los centros escolares, respecto a lo que supone la evaluación. En este sentido, señala Moreno (2011, p. 117), que el valor que ha adquirido la evaluación en las dos últimas décadas, guarda "una estrecha relación con los conceptos de calidad de la educación y accountability o rendición de cuentas".

Un buen ejemplo de ello es el Preámbulo de la LOMCE. Allí, además de exponerse que las evaluaciones externas constituyen medidas destinadas a la mejora directa de la calidad del sistema educativo, se afirma que, siguiendo las recomendaciones de la OCDE, una mayor autonomía y transparencia en la rendición de cuentas se considera necesaria para la mejora de los resultados de los centros. De hecho, Anghel, Cabrales, Sainz y Sanz (2013), en una reciente investigación muestran como en el caso de la Comunidad de Madrid, respecto al resto de Comunidades Autónomas, la publicación de los resultados de las pruebas CDI para 6을 curso 2004/2005, tuvieron, respecto a las competencias en lectura, un efecto positivo que oscilaba entre los 14 a 17 puntos PISA.

Siguiendo esta misma línea argumentativa, Marchesi (2006) nos viene a decir que dentro de la evaluación educativa nos podemos encontrar con una doble vertiente, siendo una de ellas, la rendición de cuentas y el control administrativo y la otra, la mejora del rendimiento de las escuelas. Aunque con matices, todas ellas, en nuestra opinión, confluyen en un punto, la utilización de las evaluaciones externas como factor de mejora educativa.

Esos matices a los que nos referimos, coinciden en valorar el rendimiento académico de los alumnos como un indicador fundamental de calidad educativa y se diferencian, en el objetivo último que se pretenda con el proceso evaluador. En este sentido, tomando como referente al ya citado Marchesi (2006), si se persigue la rendición de cuentas, las evaluaciones externas se vinculan a las necesidades de información por parte de la sociedad en general y de los agentes educativos en particular sobre el funcionamiento de las escuelas. Si se pretende el control administrativo, las evaluaciones externas se justifican en el necesario cumplimiento de los objetivos educativos por parte de las instituciones educativas. Por último, si el fin es la mejora de las escuelas, las evaluaciones externas se orientan a la posterior elaboración y puesta en marcha de planes internos de mejora en función de los resultados obtenidos. 
Bajo estas circunstancias, en un sistema educativo en el que hasta hace unos años la única información que recibía la comunidad educativa sobre el funcionamiento de los centros escolares, las enseñanzas que allí se impartían o el efecto que esto tenía sobre el rendimiento del alumnado, era por medio de las propias evaluaciones internas que llevaban a cabo los centros a través de los equipos de profesores, estamos de acuerdo con Gisbert (2015) en señalar que la evaluaciones externas nos son actualmente de una gran utilidad, ya que aportan un tipo de información de la que hasta hace unos años hemos carecido. Una información, por tanto, que al ser realizadas por personas $u$ organismos ajenos al funcionamiento interno de las escuelas, adquiere un gran valor al ofrecer mayor grado de objetividad, aspecto que permitirá por añadidura y según Marchesi (2006, p. 47), "la posibilidad de que los datos se interpreten a la luz de los datos obtenidos por escuelas de características similares".

En cualquier caso, lo que parece evidente es que en las últimas décadas ha habido un cambio en la cultura, en los hábitos y en la sistemática de la evaluación en los centros docentes, entre otras razones porque ya no se rinden cuentas a través de una evaluación interna. Como indica Gisbert (2015), la utilización de las evaluaciones externas modifica esa situación, ya que lleva consigo un mecanismo de control que deriva necesariamente hacia un sentimiento de responsabilidad compartida, una idea de grupo y en definitiva, a la configuración de un verdadero equipo docente, y por tanto, a nuestro parecer, hacia el desarrollo de un sentimiento de pertenencia entre el profesorado respecto a la escuela en la que desempeña su labor profesional. Por ello, consideramos que la elaboración, aplicación y posterior análisis de los resultados de las evaluaciones externas referidas al rendimiento académico de los alumnos, conllevan un compromiso conjunto de mejora de la calidad educativa a través de la revisión de planes y programas, revisión metodológica o en el mejor aprovechamiento de recursos humanos y materiales por parte de los diferentes componentes de la comunidad educativa. Todo ello, en busca de un único objetivo común, la mejora en los procesos formativos del alumnado y por tanto, de su rendimiento presente y futuro ya sea a nivel académico o profesional.

Obviamente, los cambios provocados por la institucionalización de las evaluaciones externas en los centros educativos, han traído consigo una serie de consecuencias, tanto positivas como negativas. En este sentido, señala Moreno (2011, p. 117), que entre los aspectos positivos de las evaluaciones externas "podemos mencionar una mayor aceptación de la evaluación por parte de los actores involucrados (docentes, directores, estudiantes, familias), como una tarea que forma parte de la vida de las escuelas". Asimismo, Popham (1999) destaca que en los últimos tiempos:

Ha habido importantes avances en la capacidad de la evaluación para crear nuevas pruebas estandarizadas que ofrezcan información precisa acerca del logro del alumno, lo que permite hacer inferencias válidas sobre los conocimientos y/o las habilidades que posee un alumno en un área particular de conocimiento, de modo que estos logros puedan ser comparados con una muestra nacional de alumnos de la misma edad o grado escolar.

(Moreno, 2011, p. 117). 
Por todo ello, consideramos que las evaluaciones externas, respecto al rendimiento del alumno, resultan positivas y necesarias ya que, como indica Juste (2007), generalmente pretenden conocer en qué magnitud el sistema está funcionando y si los objetivos educativos establecidos son alcanzados por más o menos alumnos, aportando por tanto, como destaca Ortiz (2012), información de diversa naturaleza que puede servir para implementar cambios en las acciones pedagógicas que se desarrollan en las aulas.

Por otro lado, son muchas las voces que se pronuncian críticas hacia las pruebas estandarizadas que se aplican en nuestro sistema educativo. A este respecto, estamos plenamente de acuerdo con Marchesi (2006) en señalar que una de las mayores dificultades que encuentran las evaluaciones externas, es el desconocimiento de las características propias de las escuelas, ya sea a nivel interno o externo respecto al contexto sociocultural en el que se ubican, así como la desconfianza que suscitan entre algunos sectores del profesorado. Este último aspecto a nuestro parecer, puede ser una de las claves para que en determinadas circunstancias, en nuestras escuelas, se desarrollen situaciones poco deseables.

A este respecto, si unimos por un lado la desconfianza o presión del profesorado al sentirse evaluado con las pruebas que analizan el rendimiento de sus alumnos y por otro, el hecho de que nos encontramos en un espacio educativo en el que cada vez más las escuelas compiten entre ellas por la captación de alumnos, entonces podemos llegar a situaciones en la que, como nos explica Juste (2007), en centros y aulas haya una modificación práctica del currículo que puede ir desde cambios en documentos de centro, como el Proyecto Educativo y Concreciones curriculares, hasta las modificaciones en las programaciones docentes. Estamos de acuerdo con el citado autor, en el hecho de que nos podemos encontrar con centros que focalicen sus recursos y sus esfuerzos en superar de manera solvente esas pruebas externas sin tener en cuenta, o llevando a un plano secundario, objetivos educativos que forman parte importante del proceso de formación integral del alumnado desvirtuándose por tanto, la función instrumental de la evaluación al tiempo que, tal como indica Shepard (2000), "impiden o expulsan valiosas prácticas de evaluación en el aula" (Moreno, 2011, p. 117).

Asimismo, en nuestra opinión, otro de los aspectos mejorables de las evaluaciones externas que miden el rendimiento académico de los alumnos, se localiza en su preferencia por valorar conocimientos, destrezas, habilidades 0 competencias vinculadas a las áreas de Lengua, Matemáticas o Ciencias, no contemplando otros aprendizajes que forman parte del proceso de aprendizaje de los alumnos y que, en la mayoría de los casos, también son parte del currículo de las diferentes etapas educativas, tales como la actividad física y el deporte, la música, las artes, las habilidades sociales, la búsqueda de información, la resolución de conflictos, las nuevas tecnologías de la información y comunicación, etc.

Además, en muchos casos, alumnos con múltiples habilidades diferentes a las evaluadas en las pruebas estandarizadas y que, desde nuestro punto de vista, resultan tan importantes como las otras para su formación e integración social, quedan señalados o favorecen, ellos mismos, que esas habilidades se vayan perdiendo en beneficio de las evaluadas, ya que desde el ámbito escolar se tiende a focalizar más la atención en ellas. 
Por último, podemos decir también que existe cierta paradoja en la aplicación de las pruebas de evaluación externa que miden el rendimiento académico de los estudiantes, ya que mientras a nivel internacional existe un creciente interés por la inclusión educativa, las pruebas estandarizadas aplicadas como herramienta de mejora de nuestros sistemas educativos tienden a olvidarse de este aspecto. Señalan Rappoport y Sandoval (2015) que si bien el principal objetivo de la inclusión educativa es la de crear una comunidad educativa que respete la diversidad del alumnado desde las diferentes perspectivas de la vida escolar, incluida la curricular, entonces las evaluaciones externas que se aplican actualmente pueden ser factores de exclusión educativa, puesto que en muchos casos los resultados y progresos de determinados grupos de alumnos no se consideran importantes, permitiéndoles eximirse de la realización de tales pruebas.

\section{A MODO DE CONCLUSIÓN}

A partir de lo expuesto a lo largo de este trabajo y valorando la importancia de las evaluaciones externas como motor de cambio y mejora de las diversas dimensiones que configuran nuestro sistema educativo, consideramos fundamental que las diferentes posturas que puedan tener los distintos agentes educativos sobre esas evaluaciones, converjan en dotarlas no tanto de un carácter sumativo como hasta ahora y sí formativo. Un carácter formativo que, entre otras cosas, facilite y haga posible la mejora de la realidad evaluada, mejorando los procesos de enseñanza y aprendizaje que se desarrollan en las aulas de nuestro país.

Como sabemos, son muchas las críticas que se ciernen sobre las evaluaciones externas por la forma en que se llevan a la práctica y su excesivo carácter cuantitativo. Por ello, abordar estas evaluaciones desde una perspectiva cualitativa, tanto desde los centros como desde las administraciones implicadas, permitirá alcanzar unos mejores resultados en futuras aplicaciones. Para conseguirlo, es imprescindible que la información que se obtenga de las evaluaciones externas respecto al rendimiento académico de los alumnos permita canalizar la toma de decisiones para la mejora de la calidad de la enseñanza.

La consecución de la tan ansiada calidad educativa, cuyos beneficiarios últimos son tanto la sociedad como el propio Estado al conseguir un mayor valor añadido entre su población, pasa irremediablemente por el nexo de unión entre ambos agentes, las escuelas. Para ello, a partir de los resultados obtenidos en las evaluaciones externas, los centros educativos a través de la elaboración de planes de mejora en los que participe la totalidad de la comunidad educativa y que se incluyan en la correspondiente Programación General Anual, deberán apostar por convertirse en organizaciones que aprenden, es decir, organizaciones flexibles para el cambio, permeables con el medio, racionales en el funcionamiento, así como colegiadas y reflexivas en sus actuaciones (Santos Guerra, 2010). Organizaciones, en definitiva, que deberán utilizar la información obtenida en las evaluaciones externas, para apostar por experiencias pedagógicas innovadoras que las permita crecer como instituciones al servicio de la sociedad y que las permita, al fin y al cabo, responder ante esa sociedad con la garantía de la calidad educativa, 
principalmente referida a los procesos de enseñanza y aprendizaje que en ellas se desarrolla.

Todo aquello que no transcurra por ese cauce de cambio y mejora no tendría justificación posible, ya que supondría que las instituciones educativas desecharían una información sistematiza y organizada, lo que nos conduciría a una inadecuada toma de decisiones respecto a algunos elementos del currículo, así como poco ajustada y realizada únicamente en función de evaluaciones internas. Evaluaciones estas que, si bien están más ajustadas a la realidad sociocultural del contexto en el que se ubica el centro, tienen el carácter subjetivo de las personas que evalúan, en este caso los equipos docentes, y por tanto repercutiría en un proceso de mejora educativa, a nuestro parecer, incompleto.

\section{REFERENCIAS}

Anghel, B., Cabrales, A., Sainz, J., y Sanz, I. (2013). Publicación de los resultados de las pruebas estandarizadas externas: ¿Tiene ello un efecto sobre los resultados escolares? Documentos de Trabajo (FEDEA), (7). Recuperado de: http://documentos.fedea.net/pubs/dt/2013/dt-2013-07.pdf

Cabrera, B. (2007). Políticas educativas en clave histórica: la LOGSE de 1990 frente a la LGE de 1970. Témpora: Revista de Historia y Sociología de la Educación, (10), 147-181.

Calero, J., y Choi, Á. (2012). La evaluación como instrumento de política educativa. Presupuesto y Gasto Público, (67), 29-42.

Delors, J. (1996). Los cuatro pilares de la educación. En Delors et al. La educación encierra un tesoro. Informe a la UNESCO de la Comisión internacional sobre la educación para el siglo XXI, (pp. 91-103). Madrid: Santillana/UNESCO.

De la Orden, A. (2009). Evaluación y calidad. Análisis de un modelo. ESE: Estudios sobre Educación, (16), 17-36

De Puelles, M. (2008). Las grandes leyes educativas de los últimos doscientos años. Participación Educativa, (7), 7-15.

De Puelles, M. (2010). Elementos de política de la educación. Madrid: UNED.

De Puelles, M. (2012). Política, legislación y educación. Madrid: UNED.

Esteve, J. M. (2009). Educación y globalización. La sociedad y la economía del conocimiento. Avances en supervisión Educativa: Revista de la Asociación de Inspectores de Educación de España, (11). Recuperado de: https://avances.adide.org/index.php/ase/article/view/430/274 
Fernández, J. M. (2008). Derecho a la educación y libertad de enseñanza en la reforma educativa (LOE). Revista de Educación, (346), 245-265.

Gisbert, X. (2015). Evaluaciones externas y calidad. Magisterio. Recuperado de: http://www.magisnet.com/noticia/21731/opinion/evaluaciones-externas-ycalidad.html

Instituto Nacional de Evaluación y Estadística y Subdirección General de Estadística y Estudios del MECD (2016). Sistema estatal de indicadores de la educación 2016. Madrid: Ministerio de Educación, Cultura y Deporte. Recuperado de: http://www.mecd.gob.es/dctm/inee/indicadores/2016/17768-sistemaestatalindicadores2016-27-32017.pdf?documentld=0901e72b824643f0

Juste, R. P. (2007). La evaluación externa y sus implicaciones: aspectos técnicos, prácticos y éticos. Avances en Supervisión Educativa: Revista de la Asociación de Inspectores de Educación de España, (6). Recuperado de: https://avances.adide.org/index.php/ase/article/view/275/235

Ley General de Educación y Financiamiento de la Reforma Educativa (LGE) (Ley 14/1970, 4 de agosto). BOE, $n^{\circ}$ 187, 6 de agosto.

Ley de Instrucción Pública (sancionada por S.M., el 9 septiembre de 1857). Recuperado de: http://www.filosofia.org/mfa/e1857ley.htm

Ley Orgánica de Calidad de la Educación (LOCE) (Ley 10/2002, 23 de diciembre). BOE, $n^{\circ}$ 307, 24 de diciembre.

Ley Orgánica de Educación (LOE) (Ley 2/2006, de 3 de mayo). BOE, nำ106, 4 de mayo.

Ley Orgánica para la mejora de la calidad educativa (LOMCE) (Ley 8/2013, 9 de diciembre). BOE, $n^{\circ}$ 295, 10 de diciembre.

Ley Orgánica de Ordenación General del Sistema Educativo (LOGSE) (Ley 1/1990, 3 de octubre). BOE, no 238, 4 de octubre.

Ley Orgánica de la participación, la evaluación y el gobierno de los centros docentes (LOPEG) (Ley 9/1995, de 20 de noviembre). BOE, $n^{\circ}$ 278, 21 de noviembre.

Ley Orgánica reguladora del Derecho a la Educación (LODE) (Ley 8/1985, 3 de julio. Texto consolidado a 30 de diciembre de 2013). BOE, no 159, 4 de julio. 
Longás, J., Civís, M., Riera, J., Fontanet, A., Longás, E., y Blanch, T. A. (2008). Escuela, educación y territorio. La organización en red local como estructura innovadora de atención a las necesidades socioeducativas de una comunidad. Pedagogía Social. Revista Interuniversitaria, (15), 137-151, doi: https://doi.org/10.7179/psri 2008.15.11

López del Castillo, Mạ. T. (2013). Historia de la inspección de primera enseñanza en España. Madrid: Ministerio de Educación, Cultura y Deporte. Marchesi, A. (2006). Cambio educativo y evaluación de las escuelas. Transatlántica de Educación, (1), 43-53.

Moreno, T. (2011). La cultura de la evaluación y la mejora de la escuela. Perfiles Educativos, 33(131), 116-130.

Orden del Consejero de Educación, por el que se aprueba el Plan General de Mejora de las Destrezas Indispensables (Orden 5420-01/2005, 18 de octubre). BOCM, no 310, 29 de diciembre.

Ortiz, F. (2012). Evaluación, impacto en el aula. Ecomatemático, 3(1), 4-10.

Plan General de Instrucción Pública (Real Decreto de 4 de agosto de 1836).

Recuperado de: http://www.filosofia.org/mfa/fae836a.htm

Plan y Reglamento de Escuelas de Primeras Letras (aprobado por S.M. en 16 de febrero de 1825). Recuperado de:

https://books.google.es/books?id=dprCeegbwvEC\&printsec=frontcover\&hl= es\&source=gbs ge summary $r \& c a d=0 \# v=0$ nepage \& $q \& f=$ false

Rappoport, S., y Sandoval, M. (2015). Inclusión educativa y pruebas estandarizadas de rendimiento. Revista de Educación Inclusiva, 8(2), 1829.

Real, C. (2012). La configuración del sistema educativo español en el siglo XIX: Legislación educativa y pensamiento político. Campo Abierto: Revista de Educación, 31(1), 69-94.

Real Decreto por el que se establecen las enseñanzas mínimas de la Educación Primaria (Real Decreto 1513/2006, 7 de diciembre). BOE, $n^{-}$ 293, 8 de diciembre.

Reglamento General de las Escuelas de Latinidad y Colegios de Humanidades (Real Cédula de S.M. y Señores del Consejo, 1826). Recuperado de: https://books.google.es/books?id=hXPLuyp46bcC\&printsec=frontcover\&hl= es\&source=gbs ge summary $r \& c a d=0 \# v=0$ nepage $\& q \& f=f a l s e$ 
Reglamento General de Instrucción Pública (Decretado por las Cortes en 29 de junio de 1821). Recuperado de: https://books.google.es/books?id=If9dlr8VUH8C\&printsec=frontcover\&hles \&source $=g$ bs ge summary $r \& c a d=0 \# v=0$ nepage $\& q \& f=f a l s e$

Reglamento Provisional de las Escuelas Públicas de Instrucción Primaria Elemental (aprobado en 1838). Recuperado de: https://books.google.es/books?id=e04fjeZ748EC\&pg=PA21\&lpg=PA21\&dq $=$ Reglamento+Provisional+de+las+Escuelas+P\%C3\%BAblicas+de+Instruc ci\%C3\%B3n+Primaria+Elemental\&source=bl\&ots=ZFOYQkMCNJ\&sig=tgX Ne5WkmUwuaj06cyU17ivz1Mg\&hl=es\&sa=X\&ved=0ahUKEwiikp Ilc NAh XKyRoKHfBOD-

Q6AEIHDAA\#v=onepage\&q=Reglamento\%20Provisional\%20de\%20las $\% 2$ OEscuelas\%20P\%C3\%BAblicas\%20de\%20Instrucci\%C3\%B3n\%20Primari a\%20Elemental\& $\mathrm{f}=$ false

Rodríguez, J. L. (2010). La evaluación en el diseño curricular. Concepto, análisis y caracterización de la evaluación en la educación española actual. En Medina, A. y Sevillano, Mạ. L. (Coords.). Diseño, desarrollo e innovación del currículum. (pp. 777-804). Madrid: Editorial Universitas.

Santamaría, R. Mํ. (2007). La política educativa en la España decimonónica y su trascendencia. Bordón. Revista de Pedagogía, 59(1), 167-176.

Santos Guerra, M. Á. (2010). La formación del profesorado en las instituciones que aprenden. Revista Interuniversitaria de Formación del Profesorado, (68), 175-200.

Tedesco, J. C. (2009). Calidad de la educación y políticas educativas. Cadernos de Pesquisa, 39(138), 795-811, doi: https://doi.org/10.1590/S0100-15742009000300006

Valle, J. M. (2012). La política educativa supranacional: un nuevo campo de conocimiento para abordar las políticas educativas en un mundo globalizado. Revista Española de Educación Comparada, (20), 109-144, doi: https://doi.org/10.5944/reec.20.2012.7595 